\title{
How Perseverance and Passion Brought Me to Canadian forestry!
}

W hen I meet new people, the second question I inevitably get (the first one being if they pronounced my name correctly), is where my name comes from. It's Dutch-I was born and raised in the Netherlands, and even obtained my degree in forestry from the Netherlands (and yes, the Netherlands does have trees...). So how did I end up in Canada, working for the Canadian Forest Service no less? Well, here's my story.

When I was a young teenager, I told my parents I wanted to live in Canada, even though at that point I had no idea I wanted to study trees. I couldn't even tell the difference between a beech and an oak. So why Canada?

As was quite common in the late 1940s, many people decided to migrate to Canada, New Zealand and other farflung places, looking for a better life and more prosperity. My great-uncle ended up in Canada, and at one point had a stint as a park ranger in Algonquin Park. We would see him seldom, but he was a great story teller, and I think one of his stories may have (subconsciously) planted the seed for a future in Canada.

But how did I end up in Forestry? I always wanted to go to vet school, but needed some extra credits, which meant an extra year of high school. During that year we had a reunion where I ran into an old classmate of mine. She asked if I was doing something with trees. And even though forestry never, ever crossed my mind of something I would be interested in, it felt like something clicked immediately. I ended getting a $\mathrm{BSc}$ in forestry, and I have literally never looked back.

What never changed was my desire to live in Canada. So when I graduated high school (but before I knew I wanted to go into forestry), I was able to spend a summer in Canada. Remember that great-uncle? Well, he has a son who lives near Belleville, and they were able to host me for a summer, which was an amazing experience. From doing tourist activities like visiting the Nia-

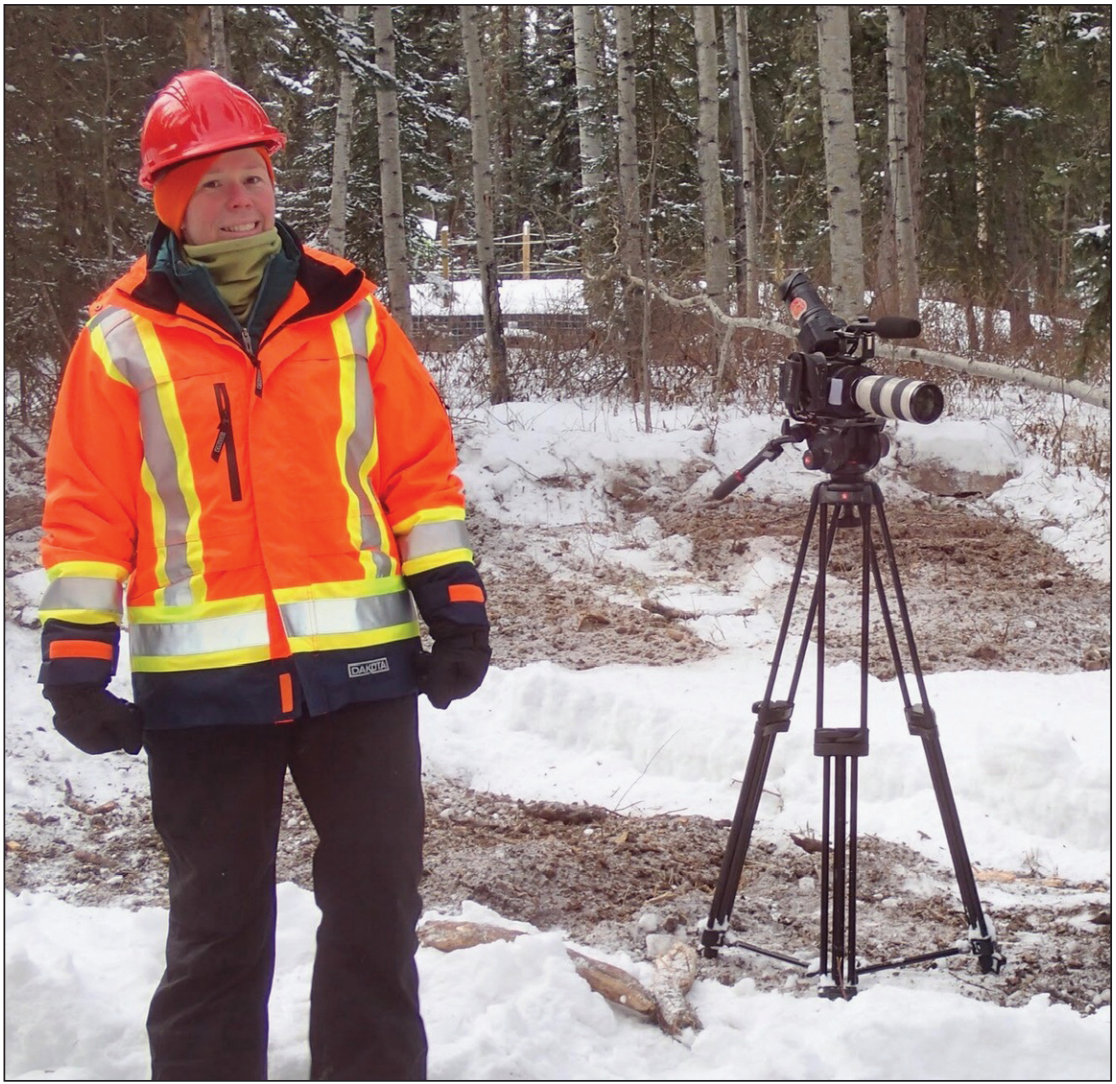

Katalijn MacAfee

gara Falls to my first-ever overnight multi-day canoe trip in Temagami, I was officially sold-Canada was where I was going to live.

I was extremely lucky that during my third year in University, I was able to spend both a semester in England and do an international placement. My best friend and I ended up spending four months working at the Petawawa Research Forest in Eastern Ontario. It was literally a dream job! We learnt so much from basic forest management to forest research activities, were involved with a prescribed burn, received basic forest fire training and were joining researchers collecting field data. I don't think we spent one day in the office.
Once I graduated, I wanted to permanently move to Canada, but that sounded easier than it actually was. I was able to secure another 6-month contract with the research forest, but had to go back to the Netherlands after that time was up. The year after, I was able to secure a one year contract (and visa), but again had to go back. I didn't qualify according to Citizenship and Immigration Canada (CIC) as a skilled worker, so wasn't able to move permanently. However, three years after graduation (and several odd-ball jobs in the Netherlands), CIC decided to lower the qualification standard and I was finally able to move and become a permanent resident! 
But at that point I didn't have a job. Luckily my cousin and his family in Belleville were super helpful, but I ended up working at Tim Hortons for a few months, simply because I had to pay my rent.

The following spring I was able to secure another short-term contract with the Research Forest, and I ended up working with a researcher to put together an annotated bibliography on white and red pine management. It ended up being a great opportunity to learn so much more about these two iconic tree species and it also provided an entry into the research world of the Canadian Forest Service. After that I had several short-term contracts, to the point where I sometimes on Friday didn't know whether I would have a job on Monday. One of these short-term contracts was working with the local logger, cutting the smaller maple and balsam while he took care of the large red pine. This was the time I met my now-husband. He still likes to jokingly say that when we met I was a real lumberjack, driving around with a chainsaw in my trunk. Admittedly, I'm actually proud of that!

During that time, there were also some changes in the overall management of the Research Forest, and the person ultimately responsible was based in Ottawa. We ended up talking at some point, and he was able to offer me another short-term stint, but this time out of the Ottawa office. This worked out quite well, since my partner lived in Ottawa. This person, George Bruemmer, ended up being absolutely instrumental for my career development. He bent-over backwards trying to keep me on, and after several contracts, a term position and several competitions, I was finally able to secure a fulltime, permanent job with the CFS. A dream come true and a huge milestone for me, since all the uncertainty and unknowns when not having a job definitely gets to you at some point. I think it's why I'm now so passionate about helping others, especially those that are trying to get a foot in the door and establishing a career. I know I wouldn't be here if I didn't have someone that believed in me, but was also not afraid to push me.

Fast-forward about 10 years and I'm still with the CFS, now in a manager's position. I love my job, but will never forget those hard, challenging and difficult first few years. I have also learned to really live life to the fullest. I have a daughter who is 7 , and we're trying to teach her as much about the outdoors as possible. She loves learning about trees and plants, and has an enormous appreciation for the world around us. It's really fun to watch.

We also decided to follow our dreams, and bought a rural property a few years ago. I have never been happier to live where we are, and show my daughter what the world around us has to offer. Even my husband, who didn't really learn much about nature until he met me, is now fully embracing our outdoor lifestyle. He's even the better tractor driver!

I know I have had a certain amount of luck in my life, but I have also been able to seek opportunities and have not been afraid to take initiative and try new things. I went from working mostly on knowledge exchange and tech transfer activities, to leading a restoration project in Alberta, simply because they were looking for someone with a silviculture background, and I told management I was interested. Which led to the further development of my career. So if I can end with one piece of advice don't be shy to show what you're capable of. Put your name forward, make sure they 'don't forget about you'. You never know what new and exciting opportunities lay ahead.

\section{Katalijn MacAfee \\ Manager, Cumulative Effects Canadian Forest Service, NRCan/RNCan}

[This article first appeared on the "Women in Wood" website at https://www.womeninwood.ca/single-post/putting-your-nameforward and is reproduced here with permission.]

\section{Comment la persévérance et la passion m'ont menée à la foresterie au Canada!}

\begin{abstract}
orsque je fais la connaissance de nouvelles personnes, la deuxième question qu'on me pose inévitablement (la première étant si elles ont prononcé correctement mon prénom), est quelle est l'origine de ce dernier. Je suis native de la Hollande où j'ai fait mes études et où j'ai obtenu mon diplôme de foresterie des Pays-Bas (et oui, il y a des arbres aux Pays-Bas...). Alors comment se fait-il que j'ai abouti au Canada et de plus à l'emploi du Service canadien des forêts? Laissez-moi vous raconter mon histoire.

Au cours de mon adolescence, j'ai déclaré à mes parents que je voulais vivre au Canada, même si à cette époque je n'avais aucune idée que je voulais étudier en foresterie. Je ne pou-
\end{abstract}

vais même pas faire la différence entre un hêtre et un chêne. Alors pourquoi le Canada?

Comme il était assez fréquent à la fin des années 1940, beaucoup de personnes songeaient à émigrer au Canada, en Nouvelle-Zélande ou dans d'autres pays lointains, à la recherche d'une meilleure vie et de revenus plus élevés. Mon grand-oncle a choisi le Canada et a trouvé une affectation temporaire au parc Algonquin en tant que garde forestier. Nous le rencontrions à l'occasion, mais c'était tout un conteur d'histoires et je crois que l'une d'entre elles a été (inconsciemment) à l'origine de ma carrière au Canada.

Mais comment ai-je abouti en fores- terie ? J'ai toujours voulu aller en médecine vétérinaire, mais il me fallait obtenir des crédits supplémentaires au secondaire, ce qui signifiait une année additionnelle d'études. Pendant cette année, nous avons eu une activité au cours de laquelle j'ai revu une ancienne compagne de classe. Elle m'a demandé si j'étudiais de près ou de loin les arbres. Même si la foresterie n'avait jamais même effleuré mon intérêt en tant que domaine d'études, j'ai senti qu'un déclic venait de s'opérer. J'allais par la suite obtenir mon baccalauréat en foresterie et je n'ai jamais remis en question mon choix.

Ce qui n’a jamais changé était mon désir de vivre au Canada. Alors après 
avoir terminé mes études secondaires (mais avant de savoir que je voulais étudier en foresterie) j'ai eu l'opportunité de passer un été au Canada. Vous vous rappelez mon grand-oncle ? Celui-ci avait un fils qui vivait près de Belleville et sa famille était prête à m'héberger pour l'été, ce qui s'est avéré être une expérience fabuleuse. Profitant de l'occasion pour faire du tourisme comme la visite des chutes Niagara, en passant par ma toute première expédition de canot s'étalant sur plusieurs jours et nuits dans la région de Temagami, je suis définitivement tombé en amour avec le Canada

J'ai eu la chance au cours de ma troisième année d'université de passer un semestre complet en Angleterre et de profiter d'un échange d'emploi à l'étranger. Ma meilleure amie et moi avons passé quatre mois à travailler à la Forêt expérimentale de Petawawa située dans l'est de l'Ontario. Ce fut un emploi de rêve ! Nous avons perfectionné nos connaissances tant sur l'aménagement forestier de base que sur les activités de recherche forestière, tout en étant impliquées dans un projet de brûlage contrôlé, en plus de recevoir une formation sur les feux de forêt et de rejoindre les chercheurs sur le terrain pour la collecte de données. Je ne me souviens pas d'avoir passé une journée entière dans un bureau.

Après avoir reçu mon diplôme, je rêvais plus que jamais d'émigrer au Canada, mais cela semblait plus facile que la réalité. J'ai été en mesure d'être employée pour une période de six mois en recherche forestière, mais j'ai dû retourner aux Pays-Bas par la suite. L'année suivante j'ai eu un contrat d'un an (et un visa), mais j'ai dû retourner en Europe. Je n'avais pas été classée comme travailleur qualifié par Citoyenneté et Immigration Canada (CIC), de telle sorte que je ne pouvais pas obtenir le statut d'immigrante. Toutefois, trois ans après l'obtention de mon diplôme (et quelques petits boulots aux PaysBas), CIC a modifié ses critères et j'ai pu finalement émigrer et devenir une résidente permanente !

Mais je n'avais toujours pas d'emploi. Par chance avec la collaboration de mon cousin et de sa famille à Belleville, j'ai pu aménager dans la région, mais j'ai dû travailler dans un Tim Hortons pendant quelques mois de façon à pouvoir couvrir mes frais d'hébergement.

$\mathrm{Au}$ cours du printemps suivant, j'ai été en mesure d'obtenir un autre contrat de courte durée avec la Forêt expérimentale et j'ai collaboré avec un chercheur à la création d'une bibliographie annotée sur l'aménagement du pin blanc et du pin rouge. Cela s'est avéré être une bonne occasion d'en apprendre plus sur ces deux espèces d'arbres iconique, tout en me permettant d'accéder au secteur de la recherche du Service canadien des forêts. J'ai obtenu par la suite quelques contrats additionnels de courte durée qui me laissaient quelques fois dans l'appréhension de ne pas savoir le vendredi ce que je ferais le lundi suivant. Au cours de l'un de ces contrats, j'ai eu à travailler avec un exploitant forestier de la région, où je devais abattre de petits érables et sapins tandis qu'il s'occupait des gros pins rouges. C'est à ce moment que j'ai fait la connaissance de celui qui est devenu mon époux. Il aime dire encore aujourd'hui en riant que lorsque nous nous sommes rencontrés, j'étais un vrai forestier, circulant en forêt avec une scie mécanique dans le coffre de ma camionnette. Certes, j'en suis effectivement fière !

À cette époque, plusieurs changements ont été apporté à la gestion générale de la Forêt expérimentale et le directeur de la celle-ci occupait dorénavant un bureau à Ottawa. Nous avons pu échanger à quelques reprises et il a été en mesure de m'offrir un autre contrat de courte durée, mais cette foisci à Ottawa. Cela s'avéra très intéressant puisque que mon conjoint vivait à Ottawa. Ce gestionnaire de la forêt, George Bruemmer, a joué un rôle déterminant dans le développement de ma carrière. Il a tiré toutes les ficelles requises pour me garder à son emploi et à la suite de quelques contrats, d'un poste à terme et de quelques concours, j'ai pu finalement décroché un emploi permanent à temps plein pour le SCF. Je réalisais enfin un rêve et j'atteignais une étape importante de ma carrière, compte tenu que l'incertitude et les inconnus qui accompagnent l'absence d'emploi finissent par vous affecter. J'estime que c'est suite à cette expérience que je suis très motivée lorsqu'il est question d'aider les autres surtout lorsqu'ils cherchent une porte d'entrée pour démarrer leur carrière. Je reconnais que je n'en serais pas là si je n'avais pu trouver une personne qui croyait en moi, mais aussi parce que je n'ai pas eu peur d'aller de l'avant.
Dix dernières années se sont écoulées depuis et me voilà toujours à l'emploi du SCF, en tant que gestionnaire d'un programme. J'adore mon emploi, mais je ne peux pas oublier les premières années difficiles, pleines de défis et de remises en question. J'ai également appris à vivre pleinement ma vie. J'ai une fille de sept ans à qui nous essayons d'inculquer autant soit peu les joies du plein air. Elle est passionnée par les arbres et les plantes, tout en ayant un très grand intérêt pour le monde qui nous entoure. C'est très réconfortant de la voir aller.

Nous avons également décidé de réaliser nos rêves par l'achat d'une propriété en milieu rural, il y a quelques années. Je n'ai jamais autant aimé vivre ailleurs que là où nous sommes établis et de montrer à ma fille ce que le monde a à nous offrir. Même mon époux, qui n'avait pas appris grand-chose sur la nature avant notre rencontre, a adopté pleinement notre mode de vie en plein air. Il est même devenu le meilleur opérateur de tracteur!

Je reconnais que j’ai bénéficier de quelques coups de chance au cours de ma vie, mais également que j'ai été en mesure de courir après les opportunités et de ne pas avoir peur de prendre les devants et d'essayer de nouvelles choses. J'ai commencé à travailler principalement dans l'échange de connaissances et des transferts technologiques, pour par la suite gérer un projet de restauration en Alberta, simplement parce que les responsables recherchaient une personne ayant une expertise en sylviculture et que j'ai dit que cela m'intéressait. Ce qui a entraîné d'autres développements dans ma carrière. Alors si je puis me permettre de conclure avec ce conseil - n'ayez pas peur de montrer ce dont vous êtes capables. Déposez votre candidature et faite en sorte de ne pas être oubliées. Vous ne savez jamais ce qui vous attend.

Katalijn MacAfee

Gestionnaire, Secrétariat des effets cumulatifs

Service canadien des forêts, RNCan/NRCan

[Cet ARTICLE A ÉTÉ PUblié PRÉCÉdeMment DANS LE SITE INTERNET « WOMEN IN WOOD » HTTPS://WWW.WOMENINWOOD.CA/SINGLE-POST/PUTTING-YOUR-NAME-FORWARD ET A ÉTÉ REPRODUIT AVEC LA PERMISSION REQUISE.] 